\title{
New criteria for selecting the origin of DNA replication in Wolbachia and closely related bacteria
}

\author{
Panagiotis Ioannidis ${ }^{1}$, Julie C Dunning Hotopp ${ }^{2}$, Panagiotis Sapountzis ${ }^{1}$, \\ Stefanos Siozios ${ }^{1}$, Georgios Tsiamis ${ }^{1}$, Seth R Bordenstein ${ }^{3}$, Laura Baldo ${ }^{4}$, \\ John H Werren ${ }^{5}$ and Kostas Bourtzis*1
}

Address: ${ }^{1}$ Department of Environmental and Natural Resources Management, University of Ioannina, 2 Seferi Street, Agrinio 30100, Greece, ${ }^{2}$ The Institute for Genomic Research, J. Craig Venter Institute, 9712 Medical Center Drive, Rockville, MD 20850, USA, ${ }^{3}$ Josephine Bay Paul Center for Comparative Molecular Biology and Evolution, The Marine Biological Laboratory, Woods Hole, MA, 02543, USA, ${ }^{4}$ Department of Biology, University of California, Riverside, USA and ${ }^{5}$ Department of Biology, University of Rochester, Rochester, USA

Email: Panagiotis Ioannidis - panioan@cc.uoi.gr; Julie C Dunning Hotopp - jdunning@jcvi.org; Panagiotis Sapountzis - psapount@cc.uoi.gr; Stefanos Siozios - ssiozios@cc.uoi.gr; Georgios Tsiamis - gtsiamis@cc.uoi.gr; Seth R Bordenstein - sbordenstein@mbl.edu; Laura Baldo - laurab@ucr.edu; John H Werren - werr@mail.rochester.edu; Kostas Bourtzis* - kbourtz@uoi.gr

* Corresponding author

Published: 20 June 2007

BMC Genomics 2007, 8:182 doi:10.1/86/147|-2164-8-182
Received: 3 November 2006

Accepted: 20 June 2007

This article is available from: http://www.biomedcentral.com/I47I-2/64/8/182

(c) 2007 loannidis et al; licensee BioMed Central Ltd.

This is an Open Access article distributed under the terms of the Creative Commons Attribution License (http://creativecommons.org/licenses/by/2.0), which permits unrestricted use, distribution, and reproduction in any medium, provided the original work is properly cited.

\begin{abstract}
Background: The annotated genomes of two closely related strains of the intracellular bacterium Wolbachia pipientis have been reported without the identifications of the putative origin of replication (ori). Identifying the ori of these bacteria and related alpha-Proteobacteria as well as their patterns of sequence evolution will aid studies of cell replication and cell density, as well as the potential genetic manipulation of these widespread intracellular bacteria.

Results: Using features that have been previously experimentally verified in the alphaProteobacterium Caulobacter crescentus, the origin of DNA replication (ori) regions were identified in silico for Wolbachia strains and eleven other related bacteria belonging to Ehrlichia, Anaplasma, and Rickettsia genera. These features include DnaA-, CtrA- and IHF-binding sites as well as the flanking genes in C. crescentus. The Wolbachia ori boundary genes were found to be hemE and COGI253 protein (CBS domain protein). Comparisons of the putative ori region among related Wolbachia strains showed higher conservation of bases within binding sites.

Conclusion: The sequences of the ori regions described here are only similar among closely related bacteria while fundamental characteristics like presence of DnaA and IHF binding sites as well as the boundary genes are more widely conserved. The relative paucity of CtrA binding sites in the ori regions, as well as the absence of key enzymes associated with DNA replication in the respective genomes, suggest that several of these obligate intracellular bacteria may have altered replication mechanisms. Based on these analyses, criteria are set forth for identifying the ori region in genome sequencing projects.
\end{abstract}




\section{Background}

Wolbachia are Gram-negative, intracellular $\alpha$-Proteobacteria that infect many invertebrates including terrestrial crustaceans, mites, spiders and filarial nematodes [1-4]. Much of the success of Wolbachia can be attributed to the diverse phenotypes they induce in hosts. These range from classical mutualism to reproductive parasitism as characterized by the ability to override chromosomal sex determination, induce parthenogenesis, selectively kill males and induce cytoplasmic incompatibility in early embryos [2-4]. The unique biology of Wolbachia has attracted a growing number of researchers interested in questions ranging from the evolutionary implications of infection to the use of this agent for pest and disease control [5-9].

These endosymbiotic bacteria are typically transmitted through the eggs of their hosts and their replication rate is regulated to avoid overgrowth prior to host reproduction [2-4]. The replication control mechanisms are not known for this growth limitation. The intensity of Wolbachia's effects has often been correlated with bacterial copy number, as reported in different host species [10-13]. A factor that may influence bacterial proliferation is the organization of the ori region. Identifying the ori of these bacteria is a key step in understanding the mechanisms of bacterial replication and for developing methods for genetic manipulation of these bacteria. Recently, the closed and annotated genomes of two Wolbachia strains have been reported $[14,15]$. However, neither of these studies identified the putative origin of replication (ori) for Wolbachia.

DNA replication in bacteria takes place by uncoiling the double stranded helix and breaking the hydrogen bonds between the complementary strands at a specific chromosomal locus, the ori region. Early events in DNA replication are subdivided into the following three steps: (i) binding of the initiator proteins to sites located within the ori region; (ii) local unwinding of the ori region; and (iii) loading of the DNA helicase and other proteins required to form the Y-shaped replication forks [16]. Typically, bacteria have a single ori region [17], although in some prokaryotes two chromosomal ori regions were experimentally identified, probably reflecting a temporal mode of DNA replication $[18,19]$.

Chromosomal replication initiates at ori, proceeds bidirectionally and terminates when the replication forks reach the termination site, terC (in the case of circular chromosomes) or the chromosome ends (in the case of linear chromosomes) [20]. The initiation of bacterial chromosome replication is mediated by the DnaA protein, which binds to specific 9-mer cis-regulatory elements called DnaA boxes located in the ori region. Usually, about ten to twenty DnaA molecules bind to five DnaA boxes and promote unwinding of the AT-rich ori region. The sequences of ori are conserved only among closely related microorganisms and vary greatly in size (from 200-1000 bp) [17]. A common feature is the presence of several DnaA boxes and an AT-rich region; a cluster of four or more DnaA boxes is indicative of a functional origin of replication [17]. In $\gamma$-Proteobacteria, the ori region is frequently located within the $r n p A-r m p H-d n a A-d n a N-r e c F-$ gyrB gene cluster and usually next to the dnaA gene [17], with the Escherichia coli ori being the most thoroughly studied [21,22].

The location of the ori region can be diverse across the bacterial lineages [23]. Among the $\alpha$-Proteobacteria, which includes Wolbachia, only the ori region of $C$. crescentus (Cori) has been experimentally identified [24]. Independent methods have provided a consistent location for Cori between hemE (CC3763) and a gene encoding a conserved hypothetical protein (CC0001) (in the present study, this gene is referred to by its NCBI COG number, COG1806) [24]. The hemE gene encodes for uroporphyrinogen decarboxylase - a component in heme biosynthesis, and the COG1806 gene has no known function but contains the conserved domain of unknown function, DUF299. The hemE/COG1806 boundary genes of Cori are present in the sequenced genomes of several other $\alpha$-Proteobacteria [25]. While Cori shows some apparent similarities with the E. coli ori such as a 40 bp AT-rich region, presence of DnaA boxes and an IHF (integration host factor) binding site, it has an additional regulatory protein, CtrA. CtrA is a global cell cycle regulator that controls $26 \%$ of the transcripts that vary during the cell cycle [26]. In vitro footprint experiments revealed five CtrA binding sites within Cori, centered over the consensus TTAA-N ${ }_{7}$-TTAA [27]. CtrA binding sites appear to be strategically organized spanning the entire length of Cori [24]. Phosphorylated CtrA binds two perfect or imperfect halves of the recognition sequence, probably as a dimer and represses chromosomal replication.

Comparison of over 30 bacterial genomes with Cori reveals a noticeable conservation of binding sites and flanking genes, although in some bacteria, chromosomal rearrangements appear to have taken place (unpublished observations). GC-skew analysis has also been used to predict the origin of DNA replication $[17,28-32]$, but it is not a good universal predictor of the ori [33], and was not predictive for the two annotated Wolbachia genomes $[14,15]$. In contrast, for the closely related Ehrlichia, Neorickettsia, and Anaplasma, GC-skew has been used to approximate the origin position in the chromosome more reliably [34-36]. However, the actual shift can occur over a fairly large region and thus this feature alone cannot reveal the precise location for the ori region. 
Given that GC-skew analysis was insufficient at predicting the ori region in several $\alpha$-Proteobacteria species, a different approach was developed. The origin of replication in C. crescentus is located between orthologs of hemE (CC_3763) and COG1806 (CC_0001), and it contains five DnaA boxes, a single binding site for IHF and five CtrA binding sites [37]. In Rickettsia prowazekii, the ori region is also located between the hemE (RP_885) and COG1806 orthologs [37].

The guidelines used by Brassinga et al. [37] for detecting Cori were applied in the present study to identify the origin of DNA replication in Wolbachia and in ten closely related $\alpha$-Proteobacteria. Computational analyses indicate that the origin of DNA replication in the $w \mathrm{Mel}$ and $w \mathrm{Bm}$ Wolbachia strains $[14,15]$ lies between a gene encoding a cystathione- $\beta$-synthase (CBS) domain protein and the Wolbachia hemE gene. The evidence relies mainly on boundary gene recognition as well as DnaA-, CtrA- and IHF-binding site identification, as described by [37]. Analysis of the corresponding sequences from an additional fifty-one Wolbachia strains supports the predicted ori as well as identifying frequent recombination at the edges of the sequences. Using the same guidelines, the ori region was also identified in the sequenced representatives of the closely related Anaplasma, Ehrlichia, Neorickettsia and Rickettsia.

\section{Results}

In silico prediction of the Wolbachia origin of replication The origin of replication of C. crescentus is located between orthologs of hemE (CC_3763) and a conserved hypothetical protein, COG1806, and contains DnaA, CtrA and IHF binding sites [37]: In $R$. prowazekii, the ori region is also located between the hemE (RP_885) and COG1806 orthologs.

Based on a slightly modified approach and findings of Brassinga et al. [37], the Wolbachia ori was predicted in silico. Our approach was based on four criteria: (a) position near either hemE or COG1806 orthologs; (b) an intergenic region (that is, the putative ori region) containing an appropriate number of binding sites for the DnaA, CtrA and IHF factors; (c) genome-wide searches confirming that no other appropriately sized intergenic region ( $>300$ bp) contains a significant number of these three characteristic binding sites and $(\mathrm{d})$ the AT-content of the predicted origins that is higher than the average for the respective genome.

First, the Wolbachia orthologs of hemE and COG1806 were searched in the recently published Wolbachia w Mel genome [15] and identified as the loci WD_1028 and WD_0341, respectively. The great distance between the two genes in Wolbachia (>600 kb) indicated no conserva- tion of the hemE-COG1806 region in Wolbachia. The lack of conservation is most likely due to a single chromosomal rearrangement resulting in two possible locations for ori:WD_0340-WD_0341 or WD_1027-WD_1028. The first region is between the heme exporter WD_0340 $(\mathrm{ccmC})$ and the COG1806 homolog (WD_0341). The second region is between the CBS domain protein WD_1027 (referred to in the present study after its NCBI COG number, COG1253) and uroporphyrinogen decarboxylase WD_1028 (hemE).

Both regions were examined for the presence of DnaA-, CtrA-, and IHF-binding sites, essential components of ori [37]. Only the intergenic region (IGR) between the COG1253 and hemE gene (position 988364-988765, 402 bp) was found to have all three characteristic binding sites. In addition, out of all 110 IGRs of greater than 300 $\mathrm{bp}$, this region has the highest number of CtrA, DnaA and IHF binding sites (eight total, $p=1 / 110=0.009$ ), the highest density of total binding domains per IGR bp $(0.020$ per bp). The next closest is the IGR between WD_0248-WD_0249, which has a much lower total number of binding sites (six) and binding site density ( 0.013 per bp). However, it lacks putative DnaA boxes, a prerequisite for an ori region. The putative ori region is one of only two IGRs $>300$ bp that have all three binding site types $(p=2 / 110=0.018)$. However, the IGR between WD_0100-WD_0102 has only 4 total binding domains (compared to 8) and, due to it's larger size (545 bp) a binding site density $1 / 3$ that of the putative ori $(0.007 v \mathrm{~s}$ $0.020)$. These data, combined with the association of the WD_1027-WD_1028 IGR with the same genes shown to be flanking the ori region in C. crescentus and R. prowazekii, provides compelling evidence that this is most likely, the origin of replication. More specifically, there are 3 DnaA, 4 CtrA and 1 IHF binding sites in the 404 bp region between COG1253 and hemE. In contrast, the region between $c c m C$ and COG1806 has only a single IHF binding site and is only $130 \mathrm{bp}$ long, which is rather short compared to the ori of related bacteria (e.g. R. prowazekii and $C$. crescentus, have replication origins $>400$ bp [37]).

Another feature of origins is that they typically contain an AT-rich region to facilitate dissociation of the DNA during replication initiation [17]. As a result, the overall AT-content of ori is higher when compared to the respective genome. Consistent with this feature, the putative $w \mathrm{Mel}$ origin is $76 \%$ AT-rich compared to the $65 \%$ average ATcontent for the genome. In addition, the ori region is significantly more AT-rich than the average found for other intergenic regions of $>300$ bp. Of 110 intergenic regions $>300 \mathrm{bp}$, only 6 have AT-content greater or equal to the ori $(p=6 / 110=0.055)$. Indeed, the AT-contents of all origins detected in this study were higher than their respective genome averages (Table 1). It has been proposed in E. coli 
that this exceptionally AT-rich DNA is the place where the initiator factor of DNA replication, the DnaA protein, first unwinds the origin $[38,39]$. These AT-rich sequences are also conspicuous because they are tandem repeats. For example, the E. coli oriC region is composed of three imperfect 13-mer sequences with the consensus sequence GATCTNTTNTTTT [38]. Similar tandem repeats were detected in all bacterial species and strains studied except in A. marginale, E. canis and N. sennetsu. In most cases the repeats were 10-12 bp long and appeared in two copies (data not shown).

These lines of in silico evidence suggest that Wolbachia has an origin of replication on its chromosome that lies between the COG1253 and hemE genes. One of the boundary genes, hemE (WD_1028), encodes for uroporphyrinogen decarboxylase - a component in heme biosynthesis. The other, COG1253 (WD_1027), encodes for a CBS domain protein. CBS domains have been shown to bind ligands with an adenosyl group such as AMP, ATP and S-AdoMet [40]. Using the publicly available HMMer program [41], it was found that this protein has a CBS domain pair and a CorC_HlyC transporter associated domain.

The putative ori regions were also identified in other complete and/or partial publicly available Wolbachia genomes by using the above mentioned criteria. The orthologs of the COG1253 (WD_1027) and hemE (WD_1028) genes were identified by BLAST searches [42] of the genomes of the wAna [43], $w$ Sim [43], $w$ Wil [44], $w$ Pip [45] and $w \mathrm{Bm}$ Wolbachia strains [14] (see Table 1). Both flanking genes are highly conserved in position and in sequence. Based on criteria set forth by Zyskind et al. [46], the regions of the $w \mathrm{Mel}$ and $w \mathrm{Bm}$ strains for which their genome sequence is available $[14,15]$, were compared indicating that: (a) 266 of 347 , or $77 \%$, of the amino acids are conserved, while 790 of 1044 (76\%) of the nucleotides are conserved in the flanking gene hemE and (b) 216 of 279, or $77 \%$, of the amino acids are conserved, while 609 of $819(74 \%)$ of the nucleotides are conserved in the flanking gene COG1253. The ori regions of the two genomes present $73 \%$ identity at the nucleotide level. The putative ori-region-related binding sites for the $w \mathrm{Ana}, w \operatorname{Sim}$ and $w$ Wil strains (all members of the A-supergroup), $w$ Pip strain (B-supergroup), and the $w \mathrm{Bm}$ strain (D-supergroup) have been depicted [see Additional Table 3]. A schematic representation is depicted in Figure 1 while the length and genome coordinates are shown in Table 1 (where applicable).

Based on the consensus sequence of the flanking genes of the putative ori region in Wolbachia [see Additional Text File 1], a PCR strategy [see Additional Figure 1 and Additional Table 2] was developed to amplify and sequence the putative ori regions of other Wolbachia strains infecting different Drosophila species: $w$ MelPop, $w \mathrm{Ri}, w \mathrm{Au}, w \mathrm{Ha}$, $w$ Yak, $w$ Tei, $w$ San, $w$ No, $w$ Ma and $w$ Mau [see Additional Table 1] [47-57]. Further sequencing of the putative ori region of an additional 37 Wolbachia strains from diverse hosts confirmed the overall characteristics of this region both in size and sequence [see Figure 1 and Additional Table 1]. Wolbachia strains belong to eight supergroups A$\mathrm{H}$ [58-61]. The present study includes representative strains from supergroups A, B, D, E and F. All strains present three DnaA and a single IHF boxes while they differ in the number of CtrA binding sites (two to seven). The sequenced putative ori regions of some Wolbachia strains present a peculiar pattern of binding sites [see Figure 1 and Additional Table 1]. It is worth noting that the pseudoscorpion Wolbachia ori does not have any CtrA binding motifs while it presents three DnaA boxes and a single IHF binding site suggesting either the existence of a very diverged CtrA binding motif (which could not be identified when compared to the consensus sequence used in the present study) or the CtrA factor may not play an important regulatory role in the DNA replication of this Wolbachia strain.

We tested for selective constraints on the binding sites for DnaA, CtrA and IHF by comparing the frequencies of polymorphic base positions in binding domain sites versus non-binding domain sites within the same IGR in different clades containing closely related Wolbachia strains (B1, B2 and A1, figure 2). The proportion of positions showing polymorphism was lower in the binding domain sites than non-binding sites for all three clades (B1 $0.08 v \mathrm{~s}$ $0.19, \mathrm{p}=0.035 ; \mathrm{B} 20$ vs $0.12, \mathrm{p}=0.001 ; \mathrm{A} 10.02$ vs $0.08, \mathrm{p}$ $=0.081$; Fisher Exact Test), with two showing significant reductions in polymorphisms within domain positions. Combining the data give 25.3 percent lower proportion of positions with polymorphisms in the binding domains relative to flanking non-binding positions $(\mathrm{p}<0.0001$ Fisher Exact Test), indicating some selective constraint on these positions.

\section{In silico prediction of the origin of DNA replication in closely related bacteria}

Applying the same criteria, the origin of DNA replication was predicted in silico for the closely related $A$. marginale St. Maries, A. phagocytophilum HZ, E. ruminantium Welgevonden, E. ruminantium Gardel, E. canis Jake and E. chaffeensis Arkansas (Table 1). Annotation, BLAST analysis and/or domain searches (using HMMer) were used to identify the two flanking genes of the putative ori regions (Table 1).

The length of the predicted ori region differs markedly by 300 bp between the two Anaplasma spp [34,36]. In A. marginale, the ori is $620 \mathrm{bp}$; in A. phagocytophilum it is just 385 
Table I: Bacterial species/strains whose partial and/or complete genome sequence was downloaded from various sources

\begin{tabular}{|c|c|c|c|c|c|c|c|c|}
\hline Organism & References & $\begin{array}{l}\text { Closed- } \\
\text { Curated } \\
\text { annotation }\end{array}$ & Genbank & Coordinates (length) & Flank One & Flank Two & $\begin{array}{l}\% \text { genome } \\
\text { AT content }\end{array}$ & $\begin{array}{l}\text { \% ori AT } \\
\text { content }\end{array}$ \\
\hline Wolbachia pipientis wMel_A & [15] & $Y$ & NC 002978 & $988364 . .988765$ (402 bp) & $\begin{array}{l}\text { WD_1027/COG } 1253 \\
\text { (CBS) }\end{array}$ & WD_1028/hemE & 64.77 & 75.87 \\
\hline Wolbachia pipientis wBm_D & [14] & $Y$ & $\underline{N C 006833}$ & 1079740..I I0 (455 bp) & $\begin{array}{l}\text { Wbm_0809/COG } 1253 \\
\text { (CBS) }\end{array}$ & Wbm_000I/hemE & 65.82 & 69.89 \\
\hline Wolbachia pipientis wAna_A & [43] & $\mathrm{N}$ & NZ AAGB00000000 & N/A (402 bp) & $\begin{array}{l}\text { WwAna0355/COGI } 253 \\
\text { (CBS) }\end{array}$ & WwAna0353/hemE & $\mathrm{N} / \mathrm{A}$ & 75.87 \\
\hline Wolbachia pipientis wSim_A & [43] & $\mathrm{N}$ & NZ AAGC00000000 & N/A (396 bp) & $\begin{array}{l}\text { WwSim049I/COG I } 253 \\
\text { (CBS) }\end{array}$ & WwSim0492/hemE & $N / A$ & 75.51 \\
\hline Wolbachia pipientis wWil_A & [44] & $\mathrm{N}$ & N/A & N/A (402 bp) & COGI253 (CBS) & hemE & $N / A$ & 75.49 \\
\hline Wolbachia pipientis wPip_B & [45] & $\mathrm{N}$ & $N / A$ & N/A (402 bp) & COGI253 (CBS) & hemE & $\mathrm{N} / \mathrm{A}$ & 79.17 \\
\hline Ehrlichia chaffeensis Arkansas & [36] & $\mathrm{Y}$ & $\mathrm{CP} 000236$ & $26939 . .2736 \mid$ (423 bp) & ECH_0030/hemE & ECH_003I/COG $/ 253$ & 69.90 & 74.70 \\
\hline Ehrlichia ruminantium Welgevonden & [35] & Y & NC 005295 & $23003 . .23424$ (422 bp) & Erum_0180/hemE & Erum_0190/COGI 253 & 72.52 & 75.19 \\
\hline Ehrlichia ruminantium Gardel & [88] & Y & NC 006831 & $4 \mid 49 . .4564(4 \mid 6$ bp $)$ & ERGA_CDS_00050/hemE & $\begin{array}{l}\text { ERGA_CDS_00060/ } \\
\text { COG } / 253\end{array}$ & 72.49 & 75.48 \\
\hline Ehrlichia canis Jake & NC_007354 & Y & NC 007354 & I I395..। | 819 (425 bp) & Ecaj_00I0/hemE & Ecaj_00II/COGI 253 & 71.04 & 73.88 \\
\hline Anaplasma marginale St. Maries & [34] & Y & NC 004842 & | | 47944..| | 48563 (620 bp) & AM_I30I/hemE & AM_1303/COG/253 & 50.24 & 63.71 \\
\hline Anaplasma phagocytophilum $\mathrm{HZ}$ & [36] & Y & $\mathrm{CP} 000235$ & 20557..2094I (385 bp) & APH_0020/hemE & APH_002I/COGI 253 & 58.36 & 71.43 \\
\hline \multirow[t]{2}{*}{ Neorickettsia sennetsu Miyayama } & [36] & Y & $\mathrm{CP} 000237$ & $855|36 . .85554|$ (406 bp) & $\begin{array}{l}\text { NSE_0967/phage } \\
\text { uncharacterized protein }\end{array}$ & NSE_0968/hemE & 58.92 & 71.92 \\
\hline & [36] & Y & $\mathrm{CP} 000237$ & $267777 . .268329$ (553 bp) & $\begin{array}{l}\text { NSE_0324/hypothetical } \\
\text { protein }\end{array}$ & NSE_0327/COG/253 & 58.92 & 58.22 \\
\hline Rickettsia typhi Wilmington & [75] & $Y$ & NC 006142 & IIIII|4..IIII496 (383 bp) & RT_0877/hemE & RT_000I/COGI806 & 71.08 & 82.25 \\
\hline Rickettsia prowazekii Madrid E & [73] & $Y$ & NC 000963 & $11111140 . .1111523$ (384 bp) & RP_885/hemE & RP_00I/COGI806 & 71.00 & 82.55 \\
\hline Rickettsia conorii Malish 7 & {$[74]$} & $Y$ & NC 003103 & $|26836| . . \mid 268755$ (395 bp) & RC_I374/hemE & RC_000I/COGI806 & 67.56 & 81.52 \\
\hline Rickettsia felis URRWXCal2 & {$[76]$} & Y & NC 007109 & |484753..|485|48 (396 bp) & RF_1400/hemE & RF_000I/COGI806 & 67.55 & 82.83 \\
\hline Caulobacter crescentus CBI5 & {$[89]$} & $Y$ & NC 002696 & $4016703 . .159$ (404 bp) & CC_3763/hemE & CC_000I/COGI806 & 32.80 & 43.56 \\
\hline
\end{tabular}

Y, yes; N, no; N/A, not applicable. 


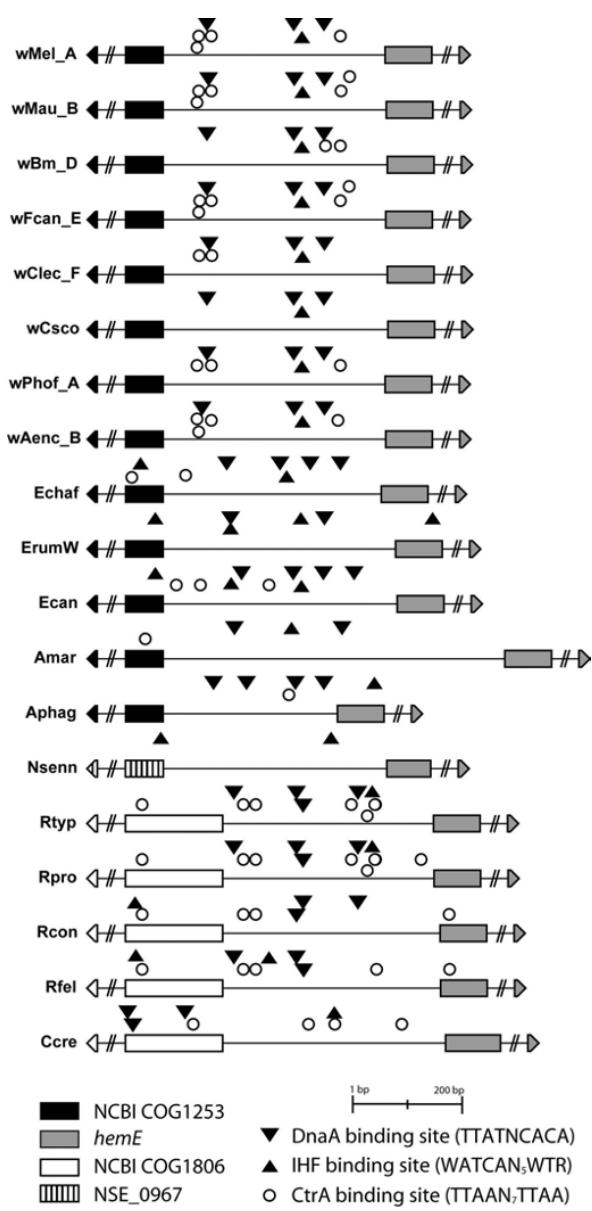

Figure I

Schematic representation of representative origins of replication. Schematic drawing of ori regions from Wolbachia, Anaplasma, Ehrlichia and Rickettsia. Inverted triangles denote DnaA boxes, circles denote CtrA binding sites and triangles indicate IHF binding sites. The flanking genes are fragmented. Some protein binding sites are located outside ori but within either of the boundary genes; however, the flanking genes were not fully sequenced from the Wolbachia strains of $F$. candida, $C$. lectularius, $C$. scorpioides, P. hoffmeyeri, and A. encedon. Note also that in $A$. marginale the ori region appears to be significantly longer due to the differently annotated hemE gene (see text for details). wMel_A, Wolbachia of Drosophila melanogaster wMel; wMau_B, Wolbachia of Drosophila mauritiana wMau; wBm_D, Wolbachia of Brugia malayi wBm; wFcan_E, Wolbachia of Folsomia candida wFcanE; wClec_F, Wolbachia of Cimex lectularius wClecF; wCsco, Wolbachia of Cordylochernes scorpioides wCsco; wPhof_A, Wolbachia of Pegoscapus hoffmeyeri wPhofA; wAenc_B, Wolbachia of Acraea encedon wAencB; Echaf, Ehrlichia chaffeensis Arkansas; ErumW, E. ruminantium Welgevonden; Ecan, E. canis Jake; Amar, Anaplasma marginale St Maries; Aphag, A. phagocytophilum HZ; Nsenn, Neorickettsia sennetsu Miyayama; Rtyp, Rickettsia typhi Wilmington; Rpro, $R$. prowazekii Madrid E; Rcon, R. conorii Malish 7; Rfel, R. felis URRWXCal2; Ccre, Caulobacter crescentus CBI5. bp (Table 1). Both species have a single CtrA binding site and a single IHF binding site that differ in both sequence and position. A. marginale has two DnaA boxes while A. phagocytophilum has four [see Figure 1 and Additional Table 1].

Origin of replication features of the different Ehrlichia spp. examined in this study are summarized in Table 1. Multiple possible IHF binding sites are found for each member (depicted in Figure 1). Of special interest is the absence of CtrA binding sites in E. ruminantium. However, if two mismatches are allowed two putative CtrA sites are found.

The putative ori region of the four different Rickettsia spp. examined here was predicted by searching for the previously described hemE/COG1806 [37] [see Figure 1, Table 1 and Additional Table 1]. Following the same type of analysis, two putative ori regions can be identified in $N$. sennetsu that are unlike other bacteria examined in this study [see Table 1 and Additional Table 1]. The first region is located between COG1806 and COG1253, is 553 bp long, and has no DnaA, no CtrA and no IHF binding site. This intergenic region contains a hypothetical gene (92 amino acids long) and a predicted tRNA-Arg. The second region is located between hemE and an uncharacterized phage protein, is $408 \mathrm{bp}$ long, and has no DnaA, no CtrA and two IHF binding sites. Although less clearly defined by the binding sites, this latter region is close to the shift in GC-skew [36] and its AT-content is higher when compared to the respective genome. Additionally, when it is compared with the first one, it appears to have more IHF binding sites in its immediate neighborhood. The above data indicate that the second region may be the putative ori region of $N$. sennetsu (Figure 1); however, it is possible that the proposed search criteria may not be appropriate for the identification of the origin of replication of N. sennetsu.

Taken together, the in silico approach can predict the putative origin of replication of most bacterial species closely related to Wolbachia including Ehrlichia, Anaplasma, and Rickettsia. In addition, the Maximum Likelihood derived trees demonstrate a clear concordance of the ori region and 16S rRNA phylogenies for the Rickettsia genus, Anaplasma/Ehrlichia genera, and Wolbachia supergroups [see Additional Figure 2].

\section{Recombination of the Wolbachia ori region}

Significant recombination events at the ori region of Wolbachia were detected within both supergroup A and B and between the two supergroups (MaxChi, $\mathrm{P}<0.001$ ). Most of the recombination breakpoints fell at one of the two edges of the intergenic region (see graph in Figure 3A). The majority of binding sites (excluding DnaA_2 and CtrA_2) occur within a region that experienced a similar 


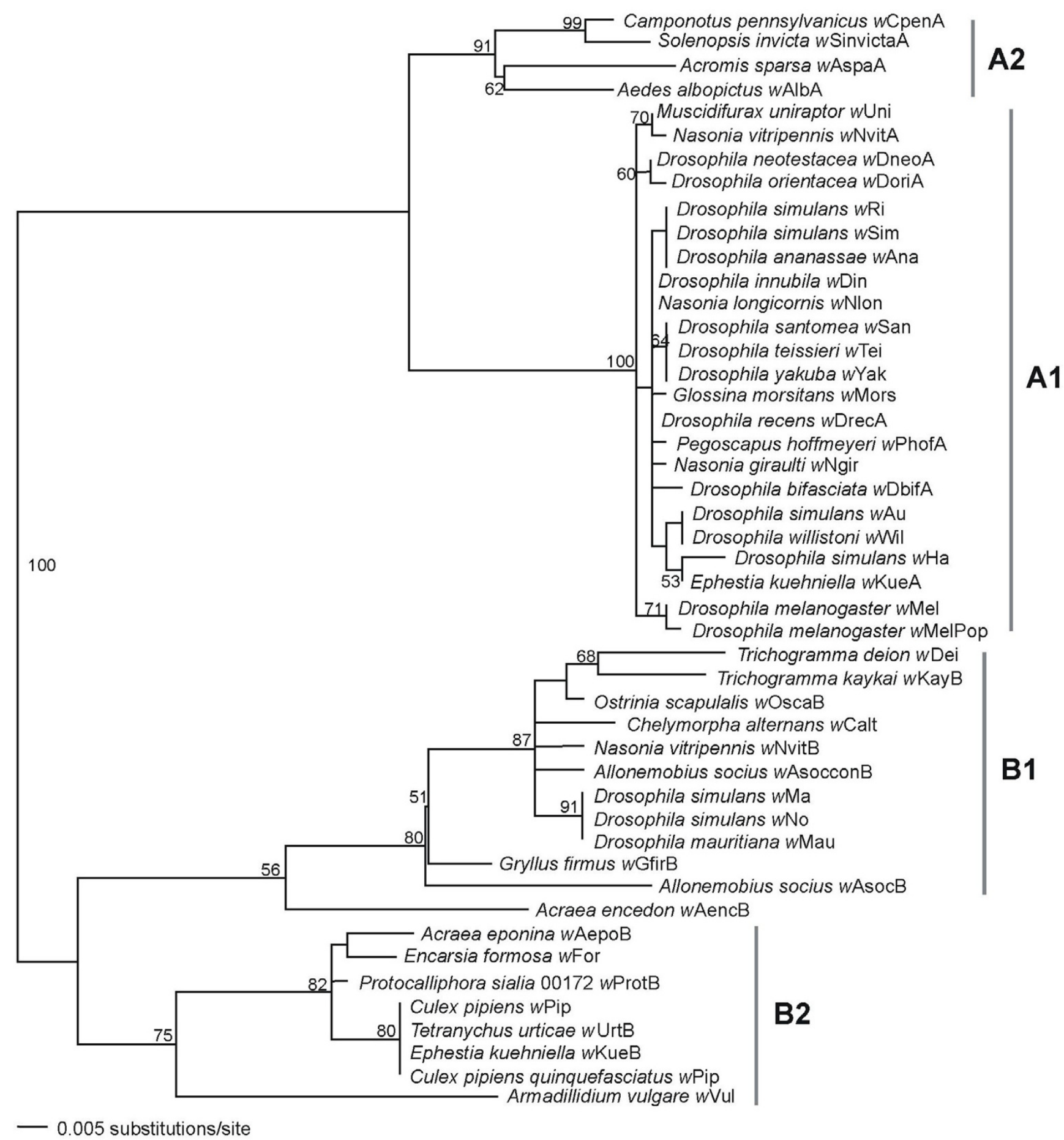

Figure 2

ori phylogeny based on 47 Wolbachia taxa. Maximum Likelihood (ML) inferred phylogeny and ML bootstrap values based on the nucleotide sequences of the ori region from the A and B Wolbachia. Subgroups are denoted AI and A2, and BI and B2. 
low number of recombination events, suggesting that usually these sites, when recombining, are exchanged as a unique sequence tract. A likely recombination breakpoint occurred in the nucleotide region between IHF_1 and DnaA_2 (see arrow, Fig. 3A) indicating a potential shuffling among binding sites at this region.

Most of the recombination breakpoints per sequence were single and thus involved recombination of sequences encompassing one of the two halves of the alignment. Clear examples of recombinant tracts can be visualized by simple examination of the shared nucleotide polymorphisms among triplets of sequences (see example in Figure $3 \mathrm{~B}$ ). For instance, the ori region of $w$ Aenc_B, a strain from the host $A$. encedon belonging to supergroup $B$, shows a clear recombinant pattern between A- and B-type sequences. Specifically, the first $210 \mathrm{bp}$ of the sequence from the wAenc_B strain shares most of the polymorphisms (70) with the B strain $w \mathrm{Ma} \_\mathrm{B}$, from D. simulans, while the remaining sequence portion shares most of polymorphisms (42) with the A strain $w$ Mel-A, from D. melanogaster. As a result, wAenc_B places as a deep branch of supergroup B, separated by a great distance from other Bstrains (bootstrap value, $\mathrm{P}=82$; data not shown); this apparent phylogenetic divergence is actually due to recombination within the sequence. A similar case of recombination is found for the $w$ Calt_B strain and involves B-type sequences. The first 500 bp of the $w$ Calt_B

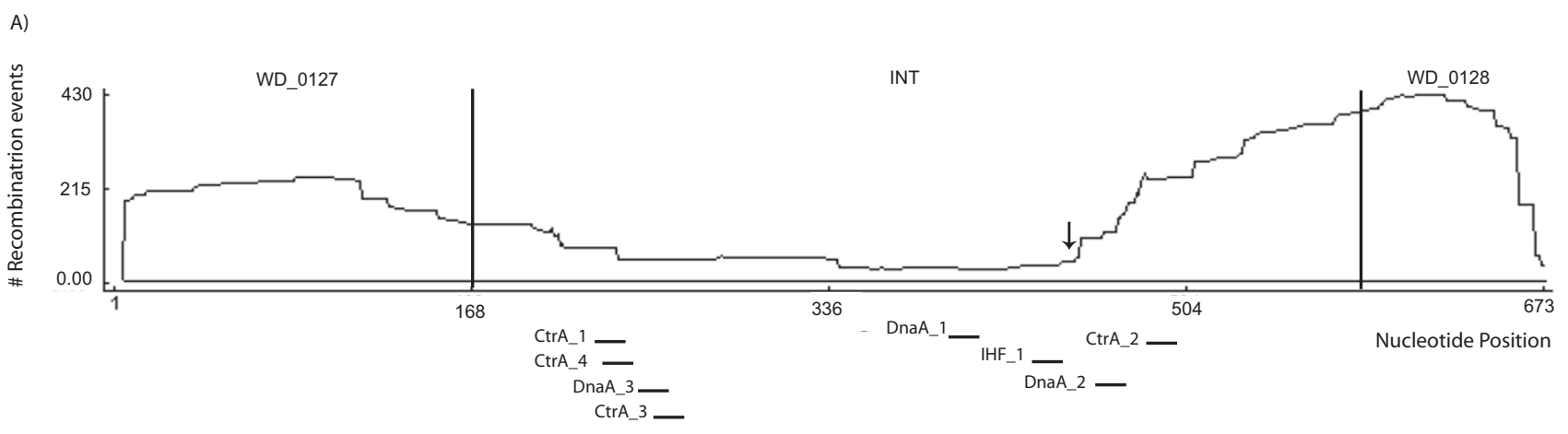

B)
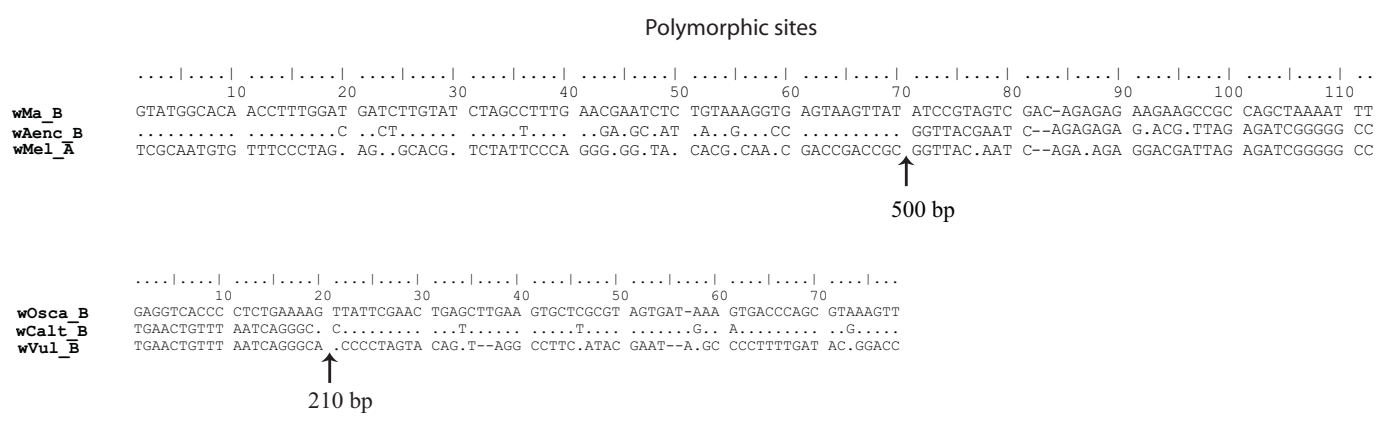

Figure 3

Recombination within Wolbachia ori sequences. Recombination results of the Wolbachia ori based on MaxChi $(\mathrm{P}<$ $0.00 \mathrm{I}$ ). (A) pattern of distribution of recombination breakpoints along the nucleotide alignment of 38 strains belonging to supergroup $A$ and $B$. The cumulative number of recombination events per site is given. The alignment includes the beginning of locus COGI 253 (WDI027, I-I68 bp), the intergenic non-coding region (INT, I69-586 bp), and the beginning of locus hemE (WDI028, 587-673 bp). Most of the breakpoints fall at the two edges of the intergenic region. Location of binding sites in the intergenic region is also shown. CtrA_I: 225-239 bp; CtrA_2: 489-503 bp (complementary strand); CtrA_3: 25I-265 bp; CtrA_4: 228-242 (complementary strand); DnaA_I: 406-4I4 bp; DnaA_2: 46I-469 bp; DnaA_3: 246-254 bp (complementary strand); IHF_I: 430-442 bp. Arrow indicates a major breakpoint. (B) Two examples of recombination among strains within supergroup $B$ and between supergroup $A$ and $B$. Only polymorphic sites are shown. For each example, putative recombination breakpoints are predicted based on the difference of shared polymophisms among the three strains and are indicated with an arrow (corresponding position in the nucleotide alignment is given). The wAenc_B Wolbachia ori sequence (belonging to supergroup $B$ ) is indicated as recombinant between the corresponding ori sequences of wMel_A (A-supergroup) and wMa_B (Bsupergroup) strains. A similar recombination event, but among supergroup $B$ strains, is observed in wCalt_B, which appears to be recombinant between wOsca_B and $w V u l \_B$. 
sequence shares most of the polymorphisms with the $w$ Vul sequence, while the following 173 bp share most polymorphisms with the $w$ OscaB sequence. Other instances of recombination involve the Wolbachia Astrains from A. albopictus, A. sparsa, C. pennsylvanicus and S. invicta, denoted A2 in figure 2. These four ori sequences are recombinant between A and B-types sequences (MaxChi, $\mathrm{P}<0.001)$.

\section{Discussion}

In the present study, we provide in silico evidence for the location of the origin of replication in Wolbachia and its close relatives: Ehrlichia, Anaplasma, Rickettsia and Neorickettsia. The analysis included fifty-three Wolbachia strains and ten additional strains from the other closely related bacterial species. All the origins predicted here are 383620 bp long. The Ehrlichia, Anaplasma, Neorickettsia, and Rickettsia predicted origins herein do align appropriately with the shift of GC-skew found in these genomes [36]. However, the actual shift occurs over a fairly large region $(\sim 20 \mathrm{kB})$ and thus is not sufficient for identifying the precise ori. The Wolbachia genomes have no clear shift in GCskew which could indicate a putative origin of replication $[14,15,17]$. The fact that Wolbachia genomes do not present a strong shift of GC-skew may be due to extensive intragenomic recombination events that in addition may have also eliminated the synteny between the genomes of the genes flanking the ori, another feature of the Wolbachia lineage. Recombination has been recently shown to be widespread across Wolbachia genomes [62]. Based on our analyses, it has clearly played a role in shaping the ori region of Wolbachia and potentially shuffled the binding sites, thus giving rise to chimeric sequences. Whether and how these DNA rearrangements have affected the replication performance in the recombinant strains remains a subject of future investigation.

\section{Prediction of origins in genomes}

As a non-coding region, the ori region is often overlooked and not annotated in genomes. However, this is a significant issue since it has an essential role in DNA replication. Therefore, we have established several criteria that should be used to identify the origin from genome sequencing data including: (a) boundary genes that are homologous to those of closely related bacteria, (b) an intergenic region 200-1000 bp in size, (c) presence of appropriate binding sites (primarily for DnaA and IHF), (d) an increased distribution of the appropriate binding sites when compared to other intergenic regions, (e) increased AT content relative to the genome, (f) increased homology to closely related sequences relative to the genome, and (g) a shift in GC-skew. Although all seven elements may not be present in all genomes, a significant combination of these elements should allow for a better prediction of this important region.

\section{The origin of replication and the CtrA, DnaA and IHF binding sites}

The presumed ori regions of the bacterial species and strains of the present study are characterized by the presence of DnaA, CtrA and IHF boxes (the exception being $N$. sennetsu). The DnaA and IHF boxes are present in all bacterial ori regions characterized. However, the CtrA boxes seem to be restricted, as yet, to the ori regions of the $\alpha$-Proteobacteria. In C. crescentus, CtrA is a global cell cycle regulator [26] and more specifically, the response regulator protein of a two-component signal transduction signal. Upon phosphorylation by a sensor histidine kinase, CtrA binds to its corresponding binding sites and represses chromosomal replication [63]. CtrA's binding sites are overlapping with a DnaA box and the IHF binding site. Thus, binding of CtrA prevents binding of DnaA and IHF [37]. CtrA is degraded before the onset of the S phase by the protease ClpXP allowing DnaA and IHF binding [64,65]. Despite A. marginale CtrA having 59\% amino acid identity with its $w \mathrm{Mel}$ ortholog, A. marginale and A. phagocytophilum ori regions have only a single CtrA binding site, as opposed to four in $w \mathrm{Mel}$. E. ruminantium has no CtrA binding site in its putative ori region while its CtrA protein is $80 \%$ identical to the A. marginale ortholog. Similarly, the putative ori regions of the pseudoscorpion C. scorpioides Wolbachia strain, E. chaffeensis, E. canis, R. conorii and the filarial nematode B. malayi Wolbachia strain $(w \mathrm{Bm})$ have zero, one, three, two and two CtrA binding sites respectively. These observations suggest that $\mathrm{CtrA}$ may be dispensable in these bacteria. Interestingly, the bacteria with zero to three CtrA binding sites in their ori region are not associated with insects. In contrast, insect-associated Wolbachia and Rickettsia bacteria usually present high number of CtrA binding sites present in their ori region ranging from four to seven (the exception being $R$. felis which has three CtrA binding sites). Whether bacterial growth control is host-dependent and is regulated through the number of the CtrA binding sites in the origin of replication region awaits experimental confirmation.

It is worth noting that there are orthologs to $d n a A, c t r A$ and $i h f A B$ in the bacterial genomes studied except $N$. sennetsu and A. marginale. They are both missing IHF- $\beta$ but have retained the IHF- $\alpha$ subunit [see Additional Table 3]. Another interesting observation is that the filarial nematode B. malayi Wolbachia strain $(w \mathrm{Bm})$ and A. phagocytophilum are missing both parA and parB, which are involved in partitioning the chromosomes [14,36]. This suggests that Wolbachia and Anaplasma replication may be quite interesting; experimental work is needed in order to clarify the roles of replication-associated proteins in binding the ori and initiating DNA replication initiation process. 
IHF binding sites were found in all bacteria using the consensus WATCAN ${ }_{5}$ WTR [37]. In some Wolbachia strains, two to three such candidate sites were found within their putative ori regions. In these cases, only the common one was retained [37]. In contrast, A. marginale and A. phagocytophilum had different IHF binding sites in both in sequence and in position. Two conserved putative IHF binding sites were detected in the putative ori region of $E$. canis and the two E. ruminantium strains, with all of them being similar both in sequence and position while a single putative IHF binding site was present in E. chaffeensis which was the only common IHF binding site found in all four Ehrlichia strains. IHF positioning varied between the four Rickettsia species. The only exception appears to be the conservation in position as well as in sequence between $R$. prowazekii and $R$. typhi. The IHF- $\beta$ subunit is missing from the genome of $A$. marginale. The only IHF identified, AM_006, is 43.2\% identical (amino acid level) to the $w$ Mel IHF- $\alpha$ subunit (WD_0057). A. phagocytophilum, all four Ehrlichia, and all four Rickettsia species have both IHF subunits. $N$. sennetsu also lacks IHF- $\beta$ [see Additional Table 3]. Whether the absence of the IHF- $\beta$ subunit is somehow correlated with the divergence/ absence of IHF binding sites in Anaplasma and Neorickettsia is not known.

\section{The evolution of the origin of replication of Wolbachia, Ehrlichia, Anaplasma, Rickettsia and Neorickettsia}

The difference in the boundary genes observed between the closely related Anaplasma, Ehrlichia, Wolbachia and the other $\alpha$-Proteobacteria is likely due to chromosomal rearrangements that have taken place in the ori region. All of the origins examined have as boundaries either the COG1253 - hemE pair (Wolbachia, Ehrlichia and Anaplasma), or the hemE - COG1806 pair (Caulobacter and Rickettsia), or the hemE - uncharacterized phage protein pair (N. sennetsu). Overlaying these observations on the phylogeny of the $\alpha$-Proteobacteria [36], the most likely ancestral configuration is that found in Caulobacter and Rickettsia. Sometime after the branching of the Anaplasmataceae, a rearrangement took place that repositioned the COG1253 gene to the position of COG1806 orthologs in Wolbachia, Ehrlichia, and Anaplasma with a second rearrangement occurring in $N$. sennetsu. The exact nature of these rearrangements cannot be determined since the synteny of the chromosomes has been lost. As discussed earlier, the distribution of ori-specific binding sites (DnaA, CtrA and IHF) is consistent with the currently accepted phylogeny of Wolbachia.

\section{Growth control, infection levels and Wolbachia-induced phenotypes}

It has been widely accepted that bacterial infection levels are positively correlated with the virulent and/or pathogenic properties of bacterial pathogens [66]. Similarly, several studies have shown that Wolbachia's ability to induce reproductive and/or virulent phenotypes is positively correlated with their intra-host infection levels [11$13,54,67]$. Furthermore, intracellular bacteria that are transmitted through the eggs of their hosts must be "prudent replicators" in order to ensure their transmission to future generations by preventing overgrowth that could lead to host death prior to reproduction. This raises the question: is the DNA replication and growth of an obligate intracellular bacterium under bacterial control, host control, phage control or some combination? Overreplication by the "popcorn" strain of Wolbachia in D. melanogaster suggests that the bacterial strain has a strong influence on replication rates within the host [54], although effects of the host also occur [12] and effects of phage have not been tested [10].

The results of the present study clearly indicate that no major differences could be detected between the ori regions of Wolbachia strains differing in ability to induce $\mathrm{CI}$, virulence, parthenogenesis or feminization. All Wolbachia strains presented the same organization in their putative origin of replication: COG1253 and hemE as boundary genes, three DnaA boxes, two to seven CtrA boxes and a single IHF binding site. A notable exception is the pseudoscorpion Wolbachia strain which presents zero CtrA binding sites. The possibility that DNA binding specificity of CtrA has changed in these bacteria is an intriguing hypothesis. CtrA contains two domains, a receiver domain at the $\mathrm{N}$-terminus and a transcriptional regulatory (DNA binding) effector domain at the C-terminus. These domains are nearly identical between $w \mathrm{Mel}$ and $w \mathrm{Bm}$ with only one positive amino acid substitution in each domain. All other changes are located at the far Cterminus where no domains are found. Since a single amino acid change can greatly affect DNA binding, it is conceivable that these two positive substitutions can alter CtrA's binding specificity. A second hypothesis is that CtrA's DNA-binding specificity did not change in $w \mathrm{Bm}$, and only the number of CtrA binding sites reduced. This CtrA binding site reduction may be associated with the mutualistic nature of the Wolbachia - B. malayi association and may have resulted in a novel control of bacterial replication. These hypotheses need to be tested experimentally.

\section{Origin of replication and genetic transformation system}

Evidence presented here for the prediction of ori location, is based on the boundary genes and on the in silico finding of characteristic DnaA, CtrA, and IHF binding sites that have been experimentally confirmed only in C. cresentus and partially in $R$. prowazekii [37]. Lack of a robust genetic transformation system for Wolbachia, Ehrlichia, Neorickettsia and most Anaplasma and Rickettsia precludes experimental verification in the bacterial species of the present 
study. However, significant progress has been made toward the development of a robust genetic transformation for A. phagocytophilum and $R$. prowazekii using homologous recombination-based and transposon-based approaches [68-70]. The fact that these bacteria (both Wolbachia and relatives) can be maintained in different cell lines $[3,71,72]$, the availability of complete genomic information [14,15,34-36,73-76] and, the presence in some of them, such as Wolbachia, of endogenous phages and insertion sequences $[77,78]$ will certainly facilitate current efforts for the genetic transformation of these intracellular bacteria.

\section{Conclusion}

We provide in silico evidence for the location of the origin of replication in Wolbachia and its close relatives: Ehrlichia, Anaplasma, Rickettsia and Neorickettsia. Putative origins of replication, which are usually 200-1000 bp long, have features in common that were used to establish a set of guidelines for properly predicting the origin in genomes. Several of the bacteria had variable sequences/ numbers of key binding sites suggesting altered modes of replication. This is supported by the lack of specific replication associated enzymes.

Intracellular bacteria that are transmitted through the eggs of their hosts are thought to be "prudent replicators" in order to ensure their transmission to future generations by not causing lethality of their host prior to reproduction. This raises the question: is the DNA replication and growth of an obligate intracellular bacterium under bacterial control, host control, phage control or some combination? The results of the present study clearly indicate that no major differences could be detected between the ori regions of Wolbachia strains inducing or non-inducing CI, being virulent or non-virulent, inducing parthenogenesis or feminization. In addition, recombination across the ori region was demonstrated for the main arthropod supergroups, A and B. Lastly and surprisingly, the origin boundary genes have changed twice in the evolution of this order of bacteria.

\section{Methods \\ DNA extraction}

Various insect hosts and Wolbachia strains were used for DNA extraction in the present study [see Additional Table 1]. Fly stocks were reared on standard corn flour - sugar yeast medium at $25^{\circ} \mathrm{C}$. Bacterial DNA was extracted using the DNeasy Tissue Kit (Qiagen) according to the manufacturer's instructions.

\section{PCR amplification and sequencing of the Wolbachia ori region}

A PCR strategy [see Results, Additional Figure 1 and Additional Table 2] was developed to amplify the ori region from the following Wolbachia strains belonging to supergroup A and $\mathrm{B}: w \mathrm{Mel}, w \mathrm{MelPop}, w \mathrm{Au}, w \mathrm{Ri}, w \mathrm{Ha}, w \mathrm{Yak}$, $w$ Tei, $w \mathrm{San}, w \mathrm{No}, w \mathrm{Ma}$ and $w \mathrm{Mau}$. Various PCR primers were used for the amplification reactions [see Additional Table 1]. PCR reaction mixtures contained $1 \times$ Taq buffer (750 mM Tris-Cl pH 8.8, $200 \mathrm{mM}\left(\mathrm{NH}_{4}\right)_{2} \mathrm{SO}_{4}, 0.1 \%$ Tween 20), $2 \mathrm{mM} \mathrm{MgCl} 2,125 \mu \mathrm{M}$ dNTPs, 12.5 pmol of each primer, 1 unit Taq polymerase (Promega) and $25 \mathrm{ng} /$ $\mu \mathrm{l}$ template DNA and were cycled with an initial denaturing step at $94^{\circ} \mathrm{C}$ for 10 minutes, 35 cycles of $94^{\circ} \mathrm{C}$ for 30 seconds, $54^{\circ} \mathrm{C}$ for 30 seconds and $72^{\circ} \mathrm{C}$ for 3 minutes followed by a final extension at $72^{\circ} \mathrm{C}$ for 10 minutes. PCR reactions were purified with Qiagen nucleotide removal kit or Qiagen gel extraction kit depending on the existence or not, of byproducts. Sequencing was performed by Macrogen (Korea). Sequence trace files from sequencing reactions were processed using the DNAStar 5.0 suite of programs.

A smaller region of ori was amplified and sequenced from a greater variety of Wolbachia strains according to the methods mentioned in [79]. Reactions to generate these smaller amplicons were attempted for 52 Wolbachia strains from almost all described supergroups using standard PCR conditions with HotStarTaq (Qiagen), according to the manufacturer's suggestions, with $0.5 \mu \mathrm{M}$ of each WD_1027_R and WD_1028_R. Reactions were initiated with a 15 minute incubation at $95^{\circ} \mathrm{C}$ followed by 50 cycles of $95^{\circ} \mathrm{C}$ for 30 seconds, $55^{\circ} \mathrm{C}$ for 30 seconds, $72^{\circ} \mathrm{C}$ for $1 \mathrm{~min}$. The primers WD_1027_R and WD_1028_R had 5 '-tags of M13 forward and reverse primer sequences, respectively, to serve as anchors for the degenerate primers in later stages of amplification and were later used for sequencing. Amplification reactions $(8 \mu \mathrm{L})$ were treated with $0.5 \mathrm{U}$ shrimp alkaline phosphatase and $1.0 \mathrm{U}$ exonuclease I (Amersham) in the supplied buffer. Sequencing reactions were performed at the J. Craig Venter Joint Technology Center (Rockville, MD) with M13 forward and reverse primers. Assembly was done with the TIGR assembler and manually curated with Cloe (ClosureEditor, a TIGR program for editing assemblies). The alignment was initially generated using CLUSTALW and curated in Bioedit v. 7.0.4.1.

The nucleotide sequences reported in this study have been deposited in GenBank under accession numbers $\underline{\mathrm{DQ} 498834}$ - DQ498882.

\section{Sequence acquisition and analysis from publicly available genomes}

Sequence information was used from publicly available genome data (GenBank) of Wolbachia and other closely related bacterial species and strains (Table 1). 


\section{In silico prediction of the ori region}

For ori-boundary identification, genes homologous to COG1253 (see results for the new proposed gene name of the CBS domain protein) and hemE were identified using BLAST. Moreover, the CBS domain protein gene was searched for conserved domains using the HMMer program [41] and the Pfam_fs hidden markov model (HMM) database.

Binding sites were identified using local perl scripts based on the previously determined consensus sequences [37]. For the CtrA binding site (TTAA- $\mathrm{N}_{7}$-TTAA), a single mismatch was allowed only in the A's and only if these mismatches followed the looser consensus TTWW- $\mathrm{N}_{7}$-TTWW described previously [37]. For the DnaA binding site, the consensus TTATNCACA was used [80]. For the IHF-binding site, the consensus WATCAN ${ }_{5}$ WTR was used $[81,82]$.

In order to examine the distribution of the CtrA-, IHF-, and DnaA-binding sites in the intergenic regions of each genome were identified using fuzznuc from the EMBOSS package [83] and locally developed scripts.

\section{Phylogenetic analysis}

The alignment for the figure 2 tree was generated using ClustalX 1.83 for Windows [84] with the default parameters and trimmed using BioEdit for Windows [85]. Maximum likelihood (ML) methods were used to infer phylogenetic relationships. Prior to ML analysis, a DNA substitution model was selected using Modeltest v3.06 and the Akaike information criterion (AIC). The selected model of evolution was based on a $382 \mathrm{bp}$ ori region with indels excluded (TVM+G). ML heuristic searches were performed using 100 random taxon addition replicates with tree bisection and reconnection (TBR) branch swapping. ML bootstrap support was determined using 100 bootstrap replicates, each using 10 random taxon addition replicates with TBR branch swapping. Searches were performed in parallel on a Beowulf cluster using a clusterpaup program and PAUP version 4.0b10 [86].

Conservation of binding domains in the Wolbachia ori region was investigated within three clades of closely related Wolbachia (B1, B2 and A1). It should be noted here that the A2 clade (see Figure 2) was not used because it is recombinant between the $\mathrm{A}$ and $\mathrm{B}$ supergroups in the ori region (see text for details). Binding site positions were identified based on a reference strain within each clade; this was straightforward due to relatively low variation within each clade. Positions were then classified as binding or non-binding. Positions within binding domains with free nucleotide designations $(\mathrm{N})$ were classified as non-binding sites. In cases where binding domains were overlapping, if both positions were $\mathrm{N}$ sites, then the position was classified as non-binding, otherwise it was classi- fied as a binding position. All positions within the IGR were then scored as polymorphic (containing at least one polymorphism) or not. Fisher Exat Tests were used to compare the proportions of polymorphic and non-polymorphic positions in binding sites versus non-binding sites.

\section{Recombination analyses}

The ori region of Wolbachia was searched for recombination signature by using the Maximum Chi Square (MaxChi2) program, implemented in the RDP2 program [87]. MaxChi is a local method that uses a sliding window approach to search for putative recombination breakpoints in a set of aligned DNA sequences. Significant discrepancies between the two partitions of the window are calculated based on the difference in the number of variable sites (VI) on either sides of the central partition. A Chisquare statistics is applied. The step size was set to 20 nucleotides and the window size set to $20 \mathrm{VI}$, gaps were included and a Bonferroni correction was applied. The highest acceptable P-value cut-off was set to 0.001 and 1000 permutation were generated. We analyzed only strains belonging to supergroup A and B and having sequences of equal length encompassing a portion of both flanking genes. The final alignment was 673 base pairs long and included 38 strains (19 A- and 19 Bstrains). The alignment was partitioned in dataset $\mathrm{A}$ and $\mathrm{B}$ (from the two supergroups) and recombination analyses were run on both single and combined datasets.

\section{Abbreviations}

ori: origin of DNA replication

CBS: Cystathione-beta-synthase domain

IHF: Integration Host Factor

IGR: Intergenic Region

\section{Authors' contributions}

PI designed primers, amplified and analyzed sequences, carried bioinformatics analysis and drafted the manuscript.

JCDH amplified and analyzed sequences across diverse Wolbachia strains, carried genomics and bioinformatics analysis, and drafted portions of the manuscript

PS designed primers, amplified and analyzed sequences across diverse Wolbachia strains.

SS amplified and analyzed sequences across diverse Wolbachia strains. 
GT amplified and analyzed sequences across diverse Wolbachia strains.

SRB designed primers, carried out the phylogenetic analysis and drafted the corresponding portion of the manuscript.

LB carried out the recombination analysis and drafted the corresponding portion of the manuscript.

JHW participated in the design of the study and coordination of research.

KB conceived of the study, participated in its design and coordination and drafted the manuscript.

All authors read and approved the final manuscript.

\section{Additional material}

\section{Additional file 1}

Additional Figure 1 - PCR strategy followed for amplifying and sequencing the ori region along with the two flanking genes of different Wolbachia strains. FRAF1 is either A-group (FRAF1MEL) or B-group (FRAF1PIP) specific and binds in slightly different positions. Click here for file [http://www.biomedcentral.com/content/supplementary/14712164-8-182-S1.jpeg]

\section{Additional file 2}

Additional Figure 2 - The Maximum Likelihood derived trees demonstrate a clear concordance of the ori region and 16S rRNA phylogenies for the Rickettsia genus (Figure 2C), Anaplasma/Ehrlichia genera (Figure $2 B$ ), and Wolbachia supergroups with one minor exception (Figure 2A). There is a weakly supported trifurcation in the 16S rRNA phylogeny (spanning supergroups $A, B$, and $E, 64 \%$ bootstrap support) that is inconsistent with the ori phylogeny ( $98 \%$ bootstrap support). Supergroups A, B, $D, E$, and $F$ are represented by sequences from $D$. melanogaster, C. pipiens, B. malayi, F. candida, and C. lectularius, respectively. The likelihood-based Shimodaira-Hasegawa (SH) test for alternative tree topologies [90] however revealed no significant difference between any of the Figure 2 topologies, specifying the ori region as a sufficient genetic marker for the broad evolutionary relationships in this intracellular clade. Selected DNA substitution models of evolution were selected using Modeltest $v 3.06$ and the Akaike information criterion (AIC). They were based on a $655 \mathrm{bp}$ ori region $(T V M+I)$ and $1377 \mathrm{bp}$ 16S rRNA sequence (HKY) for Wolbachia, a 395 bp ori region (GTR+I) and 1454 bp $16 S$ rRNA sequence $(G T R+I)$ for the Anaplasma/Ehrlichia genera, and a $382 \mathrm{bp}$ ori region $(\mathrm{HKY}+\mathrm{I})$ and $1498 \mathrm{bp} 16 \mathrm{~S}$ rRNA sequence $(\operatorname{TrN})$ for Rickettsia. ML heuristic searches were performed using 500 random taxon addition replicates with tree bisection and reconnection (TBR) branch swapping. ML bootstrap support was determined using 500 bootstrap replicates, each using 10 random taxon addition replicates with TBR branch swapping. Searches were performed in parallel on a Beowulf cluster using a clusterpaup program and PAUP version $4.0 b 10$ [86].

Click here for file

[http://www.biomedcentral.com/content/supplementary/14712164-8-182-S2.jpeg]

\section{Additional file 3}

Additional Table 1 - Wolbachia strains and closely related bacterial species used in this work. Click here for file

[http://www.biomedcentral.com/content/supplementary/14712164-8-182-S3.doc]

\section{Additional file 4}

Additional Table 2 - Primers used in this study.

Click here for file

[http://www.biomedcentral.com/content/supplementary/14712164-8-182-S4.doc]

\section{Additional file 5}

Additional Table 3 - Replication enzymes in complete genomes. Click here for file [http://www.biomedcentral.com/content/supplementary/14712164-8-182-S5.doc]

\section{Additional file 6}

Additional Text File 1 - Consensus sequence of the ori region based on which the PCR strategy presented in Additional Figure 1 and Additional Table 3 was developed.

Click here for file

[http://www.biomedcentral.com/content/supplementary/14712164-8-182-S6.fas]

\section{Acknowledgements}

We thank the following individuals for providing arthopods for this study: D. Bouchon, K. Christianson, K. Dyer, M. Hoy, S. Dobson, R. Harrison, G. Hurst, J. Jaenike, F. Jiggins, N. Lo, J. Marshall, J. Rasgon, T. Sasaki, D. Shoemaker, R. Stouthamer, M. Wade, D. Windsor, D. Zeh, and J. Zeh. We would like to thank Rahul Nene for assistance in generating sequences at TIGR and Hervé Tettelin, Stefan Oehler and Patrick Mavingui for helpful discussions and in commenting on an earlier version of the manuscript. We also thank three anonymous reviewers for helpful comments on the manuscript. PI, PS, SS, GT and KB acknowledge support of their work from intramural funding from the University of loannina. SB, JDH, LB and JW acknowledge support of their work from the U.S. National Science Foundation grant EF-0328363. SB also acknowledges the support from the NASA Astrobiology Institute (NNA04CC04A).

\section{References}

I. Stouthamer R Breeuwer JAJ, Hurst GDD: Wolbachia pipientis: microbial manipulator of arthropod reproduction. Annu Rev Microbiol 1999, 53:71-102.

2. Bourtzis K Miller TA: Insect Symbiosis. Boca Raton, FL, CRC Press; 2003.

3. O'Neill SL Hoffmann AA, Werren JH: Influential passengers: inherited microorganism and arthropod reproduction. New York, NY, Oxford University Press; 1997.

4. Werren JH: Biology of Wolbachia. Annu Rev Entomol 1997, 42:587-609.

5. Taylor MJ: Wolbachia bacteria of filarial nematodes in the pathogenesis of disease and as a target for control. Trans $R$ Soc Trop Med Hyg 2000, 94:596-598.

6. Bordenstein SR O'Hara FP, and Werren JH: Wolbachia-induced incompatibility precedes other hybrid incompatibilities in Nasonia. Nature 2001, 409:707-710.

7. Zabalou S Riegler M, Theodorakopoulou M, Stauffer C, Savakis C, Bourtzis K: Wolbachia-induced cytoplasmic incompatibility as 
a means for insect pest population control. Proc Natl Acad Sci USA 2004, I 0 I: I 5042-15045.

8. Xi Z Khoo CC, Dobson SL: Wolbachia establishment and invasion in an Aedes aegypti laboratory population. Science 2005 , 3 1 0:326-328.

9. Koukou K Pavlikaki H, Kilias J, Werren JH, Bourtzis K, Alahiotis SN: Influence of antibiotic treatment and Wolbachia curing on sexual isolation among Drosophila melanogaster cage populations. Evolution 2006, 60:87-96.

10. Bordenstein SR Marshall ML, Fry AJ, Kim U, Wernegreen JJ: The tripartite associations between bacteriophage, Wolbachia, and arthropods. PLoS Pathog 2006, 2:e43.

II. Breeuwer JA, Werren JH: Cytoplasmic incompatibility and bacterial density in Nasonia vitripennis. Genetics 1993 I35:565-574.

12. McGraw EA Merritt DJ, Droller JN, O'Neill SL: Wolbachia density and virulence attenuation after transfer into a novel host. Proc Natl Acad Sci USA 2002, 99:2918-2923.

13. Veneti Z, Clark ME, Zabalou S, Karr TL, Savakis C, Bourtzis K: Cytoplasmic incompatibility and sperm cyst infection in different Drosophila - Wolbachia associations. Genetics 2003 I 64:545-552.

14. Foster J Ganatra M, Kamal I, Ware J, Makarova K, Ivanova N, Bhattacharyya A, Kapatral V, Kumar S, Posfai J, Vincze T, Ingram J, Moran L, Lapidus A, Omelchenko M, Kyrpides N, Ghedin E, Wang S, Goltsman E, Joukov V, Ostrovskaya O, Tsukerman K, Mazur M, Comb D, Koonin E, Slatko B.: The Wolbachia genome of Brugia malayi: endosymbiont evolution within a human pathogenic nematode. PLoS Biol 2005, 3:e I 21

15. Wu M Sun LV, Vamathevan J, Riegler M, Deboy R, Brownlie JC, McGraw EA, Martin W, Esser C, Ahmadinejad N, Wiegand C, Madupu R, Beanan MJ, Brinkac LM, Daugherty SC, Durkin AS, Kolonay JF, Nelson WC, Mohamoud Y, Lee P, Berry K, Young MB, Utterback T, Weidman J, Nierman WC, Paulsen IT, Nelson KE, Tettelin $\mathrm{H}$, O'Neill SL, Eisen JA: Phylogenomics of the reproductive parasite Wolbachia pipientis wMel: a streamlined genome overrun by mobile genetic elements. PLOS Biol 2004, 2:E69.

16. Baker TA Bell SP: Polymerases and the replisome: machines within machines. Cell 1998, 92:295-305.

17. Mackiewicz P Zakrzewska-Czerwinska J, Zawilak A, Dudek MR Cebrat S: Where does bacterial replication start? Rules for predicting the oriC region. Nucleic Acids Res 2004, 32:378I-379I.

18. Robinson NP Dionne I, Lundgren M, Marsh VL, Bernander R, Bell SD: Identification of two origins of replication in the single chromosome of the archaeon Sulfolobus solfataricus. Cell 2004, I 1 6:25-38.

19. Kelman LM Kelman Z: Multiple origins of replication in archaea. Trends Microbiol 2004, 1 2:399-40I.

20. Messer W Weigel C: Initiation of chromosome replication. In Escherichia coli and Salmonella Cellular and Molecular Biology, 2nd edition Edited by: Neidhardt, FC. Washington, DC , ASM Press; 1996:1579-1601.

21. Baker TA Wickner SH: Genetics and enzymology of DNA replication in Escherichia coli. Annu Rev Genet 1992, 26:447-477.

22. Skarstad K Boye E: The initiator protein DnaA: evolution, properties and function. Biochim Biophys Acta 1994 , 1217: $111-130$

23. Tobiason DM Seifert HS: The Obligate Human Pathogen, Neisseria gonorrhoeae, Is Polyploid. PLoS Biology 2006, 4:e I85.

24. Marczynski GT Shapiro L: Control of chromosome replication in Caulobacter crescentus. Annu Rev Microbiol 2002, 56:625-656.

25. Brassinga AKC Siam R, Marczynski GT: Conserved gene cluster at replication origins of the alpha-proteobacteria Caulobacter crescentus and Rickettsia prowazekii. I Bacteriol 200I, 183:1824-1829.

26. Laub MT McAdams HH, Fraser CM, Shapiro L: Global analysis of the genetic network controlling a bacterial cell cycle. Science 2000, 290:2|44-2।48.

27. Quon KC Yang B, Domian IJ, Shapiro L, Marczynski GT: Negative control of bacterial DNA replication by a cell cycle regulatory protein that binds at the chromosome origin. Proc Natl Acad Sci USA 1998, 95:120-125.

28. Lobry JR: Asymmetric substitution patterns in the two DNA strands of bacteria. Mol Biol Evol 1996, I 3:660-665.

29. Rocha EP Danchin A, Viari A: Universal replication biases in bacteria. Mol Microbiol 1999, 32:11-16.
30. Lobry JR: A simple vectorial representation of DNA sequences for the detection of replication origins in bacteria. Biochimie 1996, 78:323-326.

31. Tillier ER Collins RA: The contributions of replication orientation, gene direction, and signal sequences to base-composition asymmetries in bacterial genomes. J Mol Evol 2000, 50:249-257.

32. Grigoriev A: Analyzing genomes with cumulative skew diagrams. Nucleic Acids Res 1998, 26:2286-2290.

33. Rocha EP: The replication-related organisation of bacterial genomes. Microbiology 2004, I50:1609-1627.

34. Brayton KA Kappmeyer LS, Herndon DR, Dark MJ, Tibbals DL, Palmer GH, McGuire TC, Knowles DP: Complete genome sequencing of Anaplasma marginale reveals that the surface is skewed to two superfamilies of outer membrane proteins. Proc Natl Acad Sci USA 2005, I 02:844-849.

35. Collins NE Liebenberg J, de Villiers EP, Brayton KA, Louw E, Pretorius $A$, Faber FE, van Heerden $H$, Josemans $A$, van Kleef $M$, Steyn HC, van Strijp MF, Zweygarth E, Jongejan F, Maillard JC, Berthier D, Botha M, Joubert F, Corton CH, Thomson NR, Allsopp MT, Allsopp BA: The genome of the heartwater agent Ehrlichia ruminantium contains multiple tandem repeats of actively variable copy number. Proc Natl Acad Sci USA 2005, I 02:838-843.

36. Dunning Hotopp JC Lin M, Madupu R, Crabtree J, Angiuoli SV, Eisen J, Seshadri R, Ren Q, Wu M, Utterback TR, Smith S, Lewis M, Khouri H, Zhang C, Niu H, Lin Q, Ohashi N, Zhi N, Nelson W, Brinkac LM, Dodson RJ, Rosovitz MJ, Sundaram J, Daugherty SC, Davidsen T, Durkin AS, Gwinn M, Haft DH, Selengut JD, Sullivan SA, Zafar N, Zhou L, Benahmed F, Forberger H, Halpin R, Mulligan S, Robinson J, White O, Rikihisa $\mathrm{Y}$, Tettelin $\mathrm{H}$.: Comparative genomics of emerging human ehrlichiosis agents. PLoS Genet 2006, 2:e2I.

37. Brassinga AKC Siam R, McSween W, Winkler H, Wood D, Marczynski GT: Conserved response regulator CtrA and IHF binding sites in the a-proteobacteria Caulobacter crescentus and Rickettsia prowazekii chromosomal replication origins. J Bacteriol 2002, I 84:5789-5799.

38. Bramhill D Kornberg A: Duplex opening by dnaA protein at novel sequences in initiation of replication at the origin of the E. coli chromosome. Cell 1988, 52:743-755.

39. Kowalski D Eddy MJ: The DNA unwinding element: a novel, cisacting component that facilitates opening of the Escherichia coli replication origin. EMBO Journal 1989, 8:4335-4344.

40. Ponting CP: CBS domains in $\mathrm{CIC}$ chloride channels implicated in myotonia and nephrolithiasis (kidney stones). J Mol Med 1997, 75:160-3.

4I. Eddy SR: Profile hidden Markov models. Bioinformatics 1998, 14:755-763.

42. Altschul SF Madden TL, Schaffer AA, Zhang J, Zhang Z, Miller W, Lipman DJ: Gapped BLAST and PSI-BLAST: a new generation of protein database search programs. Nucleic Acids Res 1997 25:3389-3402.

43. Salzberg SL Dunning Hotopp JC, Delcher AL, Pop M, Smith DR, Eisen MB Nelson WC: Serendipitous discovery of Wolbachia genomes in multiple Drosophila species. Genome Biol 2005, 6:R23

44. Salzberg SL Dunning Hotopp JC, Delcher AL, Pop M, Smith DR, Eisen $\mathrm{MB}, \mathrm{Nelson} W \mathrm{~W}$ : Correction: Serendipitous discovery of Wolbachia genomes in multiple Drosophila species. Genome Biol 2005, 6:402.

45. The Sanger Institute [http://www.sanger.ac.uk]

46. Zyskind JW Cleary JM, Brusilow WS, Harding NE, Smith DW: Chromosomal replication origin from the marine bacterium Vibrio harveyi functions in Escherichia coli: oriC consensus sequence. Proc Natl Acad Sci USA 1983, 80: I I64-II 68.

47. Hoffmann AA Turelli M, Simmons GM: Unidirectional incompatibility between populations of Drosophila simulans. Evolution | 986, 40:692-70|.

48. O'Neill SL Karr TL: Bidirectional incompatibility between conspecific populations of Drosophila simulans. Nature 1990 348: $178-180$.

49. Giordano R O'Neill SL, Robertson HM: Wolbachia infections and the expression of cytoplasmic incompatibility in Drosophila sechellia and D. mauritiana. Genetics 1995, 140:1307-1317.

50. Mercot H Llorente B, Jacques M, Atlan A, Montchamp-Moreau C: Variability within the Seychelles cytoplasmic incompatibility system in Drosophila simulans. Genetics 1995, I41:1015-1023. 
51. Rousset F Solignac M: Evolution of single and double Wolbachia symbioses during speciation in the Drosophila simulans complex. Proc Natl Acad Sci USA 1995, 92:6389-6393.

52. Bourtzis K Nirgianaki A, Markakis G, Savakis C: Wolbachia infection and cytoplasmic incompatibility in Drosophila species. Genetics 1996, 144: 1063-1073.

53. Hoffmann AA Clancy D, Duncan J: A naturally-occurring Wolbachia infection in Drosophila simulans that does not cause cytoplasmic incompatibility. Heredity 1996, 76: I-8.

54. Min K Benzer S: Wolbachia, normally a symbiont of Drosophila, can be virulent, causing degeneration and early death. Proc Natl Acad Sci USA 1997, 94:10792-10796.

55. James AC Ballard JW: Expression of cytoplasmic incompatibility in Drosophila simulans and its impact on infection frequencies and distribution of Wolbachia pipientis. Evolution Int JOrg Evolution 2000, 54:1661-1672.

56. Lachaise D Harry M, Solignac M, Lemeunier F, Benassi V, Cariou ML Evolutionary novelties in islands: Drosophila santomea, a new melanogaster sister species from Sao Tome. Proc Biol Sci 2000, 267: | 487-| 495

57. Zabalou S Charlat S, Nirgianaki A, Lachaise D, Mercot H, Bourtzis K: Natural Wolbachia infections in the Drosophila yakuba species complex do not induce cytoplasmic incompatibility but fully rescue the wRi modification. Genetics 2004, 167:827-834.

58. Bordenstein SR Rosengaus RB: Discovery of a novel Wolbachia super group in Isoptera. Curr Microbiol 2005, 5 I:393-398.

59. Lo N Casiraghi M, Salati E, Bazzocchi C, Bandi C: How many wolbachia supergroups exist? Mol Biol Evol 2002, 19:34I-346.

60. Werren JH Zhang W, Guo LR: Evolution and phylogeny of Wolbachia: reproductive parasites of arthropods. Proc Biol Sci 1995, 261:55-63.

61. Rowley SM Raven RJ, McGraw EA: Wolbachia pipientis in Australian spiders. Curr Microbiol 2004, 49:208-2I4.

62. Baldo L Bordenstein SR, Wernegreen IJ, Werren JH: Widespread recombination throughout Wolbachia genomes. Mol Biol Evol 2006, 23:437-449.

63. Reisenauer A Quon K, Shapiro L: The CtrA response regulator mediates temporal control of gene expression during the Caulobacter cell cycle. I Bacteriol 1999, 181:2430-2439.

64. Domian II Quon KC, Shapiro L: Cell type-specific phosphorylation and proteolysis of a transcription regulator controls the GI-to-S transition in a bacterial cell cycle. Cell 1997, 90:4I5-424.

65. Jenal $U$ Fuchs $T$ : An essential protease involved in bacterial cell-cycle control. EMBO J 1998, 19:5658-5669.

66. Ewald PW: Evolution of Infectious Disease. Oxford, Oxford University Press; 1994.

67. Mouton L Dedeine F, Henri H, Bouletreau M, Profizi N, Vavre F: Virulence, multiple infections and regulation of symbiotic population in the Wolbachia-Asobara symbiosis. Genetics 2004 168:181-189.

68. Rachek LI Tucker AM, Winkler HH, Wood DO: Transformation of Rickettsia prowazekii to rifampin resistance. J Bacteriol 1998 I80:2| |8-2|24.

69. Qin A Tucker AM, Hines A, Wood DO: Transposon mutagenesis of the obligate intracellular pathogen Rickettsia prowazekii. Appl Environ Microbiol 2004, 70:2816-2822.

70. Felsheim RF HMJ Nelson CM, Burkhardt NY, Barbet AF, Kurtti TJ, Munderloh UG: Transformation of Anaplasma phagocytophilum. BMC Biotechnol 2006, 31 : 42.

71. Raoult D Brouqui P: Rickettsiae and Rickettsial diseases at the turn of the third millenium. Paris, Elsevier; 1999.

72. Dobson SL Marsland EJ, Veneti Z, Bourtzis K, O'Neill SL: Characterization of Wolbachia host cell range via the in vitro establishment of infections. Appl Environ Microbiol 2002, 68:656-660.

73. Andersson SG Zomorodipour A, Andersson JO, Sicheritz-Ponten T, Alsmark UC, Podowski RM, Naslund AK, Eriksson AS, Winkler HH, and Kurland CG: The genome sequence of Rickettsia prowazekii and the origin of mitochondria. Nature 1998 396: $133-140$.

74. Ogata $H$ Audic $S$, Renesto-Audiffren $P$, Fournier PE, Barbe $V$, Samson D, Roux V, Cossart P, Weissenbach J, Claverie JM, Raoult D: Mechanisms of evolution in Rickettsia conorii and Rickettsia prowazekii. Science 200I, 293:2093-2098.

75. McLeod MP Qin X, Karpathy SE, Gioia J, Highlander SK, Fox GE, McNeill TZ, Jiang H, Muzny D, Jacob LS, Hawes AC, Sodergren E, Gill
R, Hume J, Morgan M, Fan G, Amin AG, Gibbs RA, Hong C, Yu XJ, Walker DH, Weinstock GM.: Complete genome sequence of Rickettsia typhi and comparison with sequences of other Rickettsiae. J Bacteriol 2004, 186:5842-5855.

76. Ogata $H$ Renesto P, Audic S, Robert C, Blanc G, Fournier PE, Parinello $\mathrm{H}$, Claverie JM, Raoult $D$ : The genome sequence of Rickettsia felis identifies the first putative conjugative plasmid in an obligate intracellular parasite. PLOS Biology 2005, 3:e248.

77. Masui S Kamoda S, Sasaki T, Ishikawa H: The first detection of the insertion sequence ISWI in the intracellular reproductive parasite Wolbachia. Plasmid 1999, 42:13-19.

78. Masui S Kamoda S, Sasaki T, Ishikawa H: Distribution and evolution of bacteriophage WO in Wolbachia, the endosymbiont causing sexual alterations in arthropod. I Mol Evol 2000 5 I:49|-497.

79. Baldo L HJC Jolley KA, Bordenstein SR, Biber SA, Choudhury RR, Hayashi C, Maiden MC, Tettelin $\mathrm{H}$, Werren JH: Multilocus sequence typing system for the endosymbiont Wolbachia. Appl Environ Microbiol 2006, 72:7098-7I I 0 .

80. Fuller RS Funnell, BE, Kornberg, A: The dnaA protein complex with the E. coli chromosomal replication origin (oriC) and other DNA sites. Cell 1984, 38:889-900.

81. Craig NL Nash HA: E. coli integration host factor binds to specific sites in DNA. Cell 1984, 39:707-716.

82. Goodman SD Velten NJ, Gao Q, Robinson S, Segall AM: In vitro selection of integration host factor binding sites. J Bacteriol 1999, I 8 I:3246-3255.

83. Rice P Longdon I, Bleasby A: EMBOSS: The European Molecular Biology Open Software Suite. Trends Genet 2000, 16:276-277.

84. Thompson JD Gibson TJ, Plewniak F, Jeanmougin F, Higgins DG: The ClustalX windows interface: flexible strategies for multiple sequence alignment aided by quality analysis tools. Nucleic Acids Res 1997, 25:4876-4882.

85. Hall TA: BioEdit: a user-friendly biological sequence alignment editor and analysis program for Windows 95/98/NT. Nucl Acids Symp Ser 1999, 41:95-98.

86. Swofford DL: PAUP*: Phylogenetic analysis using parsimony (* and other methods), version 4.0bl0. Sunderland, MA, Sinauer Associates; 2003.

87. Martin DP Williamson C, Posada D: RDP2: recombination detection and analysis from sequence alignments. Bioinformatics 2005, $21: 260-262$

88. Frutos R Viari A, Ferraz C, Morgat A, Eychenie S, Kandassamy $Y$, Chantal I, Bensaid A, Coissac E, Vachiery N, Demaille J, Martinez D Comparative genomic analysis of three strains of Ehrlichia ruminantium reveals an active process of genome size plasticity. J Bacteriol 2006, I 88:2533-2542.

89. Nierman WC Feldblyum TV, Laub MT, Paulsen IT, Nelson KE, Eisen JA, Heidelberg JF, Alley MR, Ohta N, Maddock JR, Potocka I, Nelson WC, Newton A, Stephens C, Phadke ND, Ely B, DeBoy RT, Dodson RJ, Durkin AS, Gwinn ML, Haft DH, Kolonay JF, Smit J, Craven MB, Khouri H, Shetty J, Berry K, Utterback T, Tran K, Wolf A, Vamathevan J, Ermolaeva M, White O, Salzberg SL, Venter JC, Shapiro L, Fraser CM: Complete genome sequence of Caulobacter crescentus. Proc Natl Acad Sci USA 200I, 98:4I36-4I4I.

90. Shimodaira H Hasegawa M: Multiple comparisons of log-likelihoods with applications to phylogenetic inference. Mol Biol Evol 1999, 16: II|4-1116. 